\title{
Neuronal Expression of Synaptotagmin-Related Gene 1 Is Regulated by Thyroid Hormone during Cerebellar Development
}

\author{
Gregory B. Potter, Fabrizio Facchinetti, Gerard M. J. Beaudoin III, and Catherine C. Thompson \\ Kennedy Krieger Research Institute and Department of Neuroscience, Johns Hopkins University School of Medicine, \\ Baltimore, Maryland 21205
}

Thyroid hormone (TH) is essential for proper brain development, acting through nuclear receptors that modulate the expression of specific genes in response to hormone binding. In a screen for genes regulated by $\mathrm{TH}$ in the rat cerebellum, we recently identified a novel gene, synaptotagmin-related gene 1 (Srg1). The Srg1 protein is structurally similar to synaptotagmins, a family of proteins involved in regulating neurotransmission. To elucidate a potential role of Srg1 in brain development, we have investigated the developmental and $\mathrm{TH}$-regulated expression of Srg1 in the neonatal rat brain. We show that expression of both Srg1 RNA and protein is detected only in the brain and specifically in neurons. Srg1 mRNA and protein levels increase postnatally, nearing adult levels after the third postnatal week. Neonatal TH deficiency results in a significant reduction and delay in expression of both Srg1 RNA and protein.

Multiple genetic and epigenetic factors contribute to the formation and function of the mammalian CNS. One such epigenetic factor, thyroid hormone (TH), is required for proper development of the mammalian brain. Insufficient TH during perinatal development adversely affects a number of developmental processes, including neuronal migration and differentiation, synaptogenesis, and myelination (Eayrs, 1971; Morreale de Escobar et al., 1983; Schwartz, 1983). The effects of TH deficiency have been well documented in the cerebellum, which shows retarded cell differentiation, altered Purkinje cell structure, and reduced synapse number (Nicholson and Altman, 1972a,b) (for review, see Koibuchi and Chin, 2000).

The effects of $\mathrm{TH}$ are mediated through the action of nuclear receptor proteins. $\mathrm{TH}$ receptors bind to specific DNA sequences and subsequently activate or repress the transcription of nearby genes in response to hormone binding (Mangelsdorf et al., 1995; Zhang and Lazar, 2000). Therefore, to understand the mechanism by which TH mediates developmental processes, it is critical to identify specific changes in gene expression induced by $\mathrm{TH}$ in the developing brain. Despite the importance of $\mathrm{TH}$ for brain development, a limited number of genes regulated by TH in the brain have been identified (for review, see Oppenheimer and Schwartz, 1997; Thompson and Potter, 2000). We have shown

Received Jan. 18, 2001; revised March 28, 2001; accepted March 29, 2001.

This work was supported by the John Merck Fund and by National Institutes of Health Grant DK46074. We thank S. Chen and O. Martin for technical assistance and Drs. J. Pevsner and M. Blue for helpful discussions.

G.B.P. and F.F. contributed equally to this work.

Correspondence should be addressed to Dr. Catherine C. Thompson, Kennedy Krieger Research Institute, 4th floor, 707 North Broadway, Baltimore, MD 21205. E-mail: thompsonc@kennedykrieger.org.

F. Facchinetti's present address: Research and Innovation Biotechnologies, Padova, 35127 Italy.

Copyright (C) 2001 Society for Neuroscience $\quad 0270-6474 / 01 / 214373-08 \$ 15.00 / 0$
Using immunohistochemistry, we were able to detect Srg1 protein in numerous brain regions. In the cerebellum, Srg1 protein is localized to the molecular layer, indicating that it is highly expressed in granule cell axons. To further examine Srg1 expression in cerebellar granule cells (CGCs), we used an in vitro cell culture model. In primary cultures of CGCs, Srg1 expression is significantly reduced in the absence of TH. Srg1 mRNA is rapidly upregulated in cultured CGCs, suggesting a direct response to $\mathrm{TH}$. Neuronal and TH-regulated expression of Srg1, together with its localization to neurites, implicates Srg1 as an important component of the program of gene expression induced by $\mathrm{TH}$ in the developing brain.

Key words: thyroid hormone; development; cerebellum; nuclear receptor; gene expression; synaptotagmin

that expression of synaptotagmin-related gene 1 ( $\mathrm{Srg} 1)$ is induced by $\mathrm{TH}$ in the developing cerebellum, suggesting that Srg1 has an important role in neural development (Thompson, 1996). Consistent with this idea, $\operatorname{Srg} 1 \mathrm{mRNA}$ is preferentially expressed in the brain and is maximal at a time in development when TH is known to be critical [postnatal day 7 (P7) to P21 in the rat] (Thompson, 1996).

Srg1 cDNA encodes a protein related to synaptotagmins, a family that includes at least 12 distinct mammalian genes (for review, see Schiavo et al., 1998). Synaptotagmin I (SytI) was originally identified as a component of synaptic vesicles and was subsequently shown to participate in $\mathrm{Ca}^{2+}$-mediated exocytosis (Geppert et al., 1994; Bajjalieh and Scheller, 1995; Südhof and Rizo, 1996). Srg1 shares a similar overall structure with synaptotagmins, consisting of an N-terminal transmembrane domain and tandem domains related to the regulatory region of protein kinase $\mathrm{C}$ (C2 domains) (Nalefski and Falke, 1996; Rizo and Südhof, 1998). Although the function of synaptotagmin I has been studied in great detail, the function of other synaptotagmins remains unknown.

Regulation of Srg1 expression by $\mathrm{TH}$ during brain development, together with the structural similarity of $\operatorname{Srg} 1$ to a protein known to be important for brain function, implicates Srg1 as a critical component of the developing brain. To help ascertain a potential role of Srg1, we have analyzed the expression and localization of Srg1 mRNA and protein during development, both in normal and TH-deficient rats. We find that TH deficiency causes a striking delay in the expression of both Srg1 mRNA and protein in the developing brain. In the cerebellum, Srg1 is highly expressed in the axons of mature, differentiated granule cells. Given the effects of $\mathrm{TH}$ deficiency on synaptic structure and function, $\mathrm{Srg} 1$ is a candidate for mediating these effects of TH on neuronal development. 


\section{MATERIALS AND METHODS}

Animal care. Timed pregnant Sprague Dawley rats were purchased from Charles River Laboratories (Wilmington, MA). Starting at day 13 of gestation, animals were treated with $0.025 \%$ methimazole (Sigma, St. Louis, MO) in their drinking water to induce TH deficiency in their pups. We have used this method previously to reduce $\mathrm{TH}$ levels and $\mathrm{TH}-$ responsive gene expression in the neonatal rat brain (Thompson, 1996). Animal care and treatment were in accordance with National Institutes of Health Guidelines and were approved by the Johns Hopkins University School of Medicine Institutional Animal Care and Use Committee.

Antibody production. Srg1 protein was expressed as a fusion with the bacterial protein trpE by subcloning a $1.2 \mathrm{~kb}$ Bam HI fragment of the Srg1 cDNA [encoding amino acids (aa) 208-421] into pATH21. Protein was induced with indoleacrylic acid in bacterial strain DH5 and purified by SDS-PAGE. Acrylamide containing the protein was injected into New Zealand White rabbits (Covance Research Products, Inc., Denver, PA). The antiserum was affinity purified using a bacterially expressed glutathione-S-transferase (GST)-Srg1 fusion protein produced by cloning the same cDNA fragment into pGEX3X (Amersham Pharmacia Biotech, Piscataway, NJ). The antiserum was tested for specificity both for immunohistochemistry and Western blot analysis. No signal was detected when primary antiserum was omitted or in the presence of a specific competitor protein (see Figs. $1 B, 4 B$ ). For competition experiments, proteins [GST, GST-synaptotagmin I (expression plasmid kindly provided by J. Pevsner, Kennedy Krieger Institute), and GST-Srg1] were produced as bacterial fusion proteins. The concentration of fusion proteins was estimated by Coomassie blue staining of extracts run on SDS-polyacrylamide gels. Equal amounts of competitor proteins were added along with primary antiserum.

$R N A$ preparation/Northern blot analysis. For the developmental Northern blot analysis, pups from at least two independent, equal-size litters were used. Each point represents RNA prepared from pooled tissue of four animals (equal number of males and females) from different litters. For tissue distribution, equal numbers of male and female adult rats were used. Animals were killed by decapitation, and tissues were removed and frozen on dry ice. Total RNA was prepared as described previously (Chomczynski and Sacchi, 1987). For Northern blot analysis, RNA samples $(15 \mu \mathrm{g} / \mathrm{lane})$ were separated on agarose-formaldehyde gels and transferred to nitrocellulose. Ethidium bromide staining was used to ensure that equivalent amounts of RNA were loaded per lane. ${ }^{32} \mathrm{P}$ labeled cDNA probes were prepared by random priming. For quantitation of the time-course experiment, blots were scanned using a Fuji BAS-2500 phosphorimager with Image Gauge (version 3.3) software (Fujifilm, Tokyo, Japan). Values were normalized to the values obtained using a cDNA probe for glyceraldehyde-3-phosphate dehydrogenase on the same blot. Results are the average of two independent experiments. The probe for Srg1 was from nucleotide (nt) position 253-2071 of the rat Srg1 cDNA (Thompson, 1996). Isoform-specific probes for rat synaptotagmins I and IV were made by PCR amplification from a rat brain cDNA library using the following primers: SytI (Perin et al., 1990), 5'-GAGCGGAGGCAGCGAGAGTACTC-3', 5'-CTTAAGGGCCTGATCCTTCATG-3'; SytIV (Hilbush and Morgan, 1994; Ullrich et al., 1994; Vician et al., 1995), 5'-CATGGCTCCTATCACCACCAG-3', 5'-AAGTGCTGGACTTCAAGCACTCAG-3'.

Western blot analysis. Blots were blocked with 3-5\% nonfat dry milk in PBS solution and then incubated with primary antibody diluted in blocking solution. Affinity-purified Srg1 antiserum was used at a dilution of 1:100. Other primary antibodies and relative dilutions were as follows: monoclonal mouse anti-synaptotagmin I, 1:100 (kindly provided by Dr. R. Mains, University of Connecticut Health Center, Farmington, CT); glial fibrillary acidic protein (GFAP), 1:250 (Sigma); tubulin, 1:3000 (Sigma); and actin, 1:250 (Sigma). Immunoreactive proteins were identified by enhanced chemiluminescence (ECL) under conditions specified by the manufacturer (Amersham Pharmacia Biotech). For the developmental analysis, pups from two independent, equal-size litters were used. Tissue was pooled from an equal number of male and female animals, and $\sim 60 \mu \mathrm{g}$ of total protein was used for each point. The experiment was done twice with the same result, and Figure 3 as shown is a representative Western blot. For tissue distribution, extracts were prepared from individual adult rats. The same result was obtained with male and female animals.

Cell culture. Primary cultures of cerebellar granule cells (CGCs) were generated from 8-d-old Sprague Dawley rat pups as described previously (Facchinetti et al., 1998). Cells were plated at a density of $2.5 \times 10^{-5}$ cells $/ \mathrm{cm}^{2}$ and maintained in basal Eagle's medium containing $10 \%$ fetal calf serum, $25 \mathrm{~mm} \mathrm{KCl}, 2 \mathrm{~mm}$ glutamine, and $50 \mu \mathrm{g} / \mathrm{ml}$ gentamycin. After
18-20 hr, cytosine arabinoside $(10 \mu \mathrm{M})$ was added to the culture media to halt non-neuronal cell proliferation. To obtain cerebellar mixed glial cultures, cells were shifted to minimal essential medium (MEM) containing $10 \%$ fetal calf serum, $6 \mathrm{mg} / \mathrm{ml}$ glucose, $2 \mathrm{~mm}$ glutamine, and 50 $\mu \mathrm{g} / \mathrm{ml}$ gentamycin 16-18 hours after plating. Culture media were changed every $2 \mathrm{~d}$ until confluency, resulting in a monolayer consisting primarily of astrocytes and oligodendrocyte precursors. Primary mixed glial cultures were prepared from cortices obtained from postnatal day 1 Sprague Dawley rats and maintained in MEM containing $10 \%$ fetal calf serum, $6 \mathrm{mg} / \mathrm{ml}$ glucose, $2 \mathrm{~mm}$ glutamine, and $50 \mu \mathrm{g} / \mathrm{ml}$ gentamycin. To deplete serum of L-3,5,3'-triiodothyronine (L-T3) and L-thyroxine, treatment with AG1-X8 resin (Bio-Rad, Hercules, CA) and charcoal (Sigma) was performed as described previously (Samuels et al., 1979). To restore physiological levels of TH, L-T3 (Sigma) was added to a final concentration of $10^{-7} \mathrm{M}$.

Immunohistochemistry. For immunohistochemical staining of rat brain, sections were prepared from the cerebellum at postnatal day 12 or 16 . Rats were deeply anesthetized by injection of $1 \mathrm{mg} / \mathrm{gm}$ body weight chloral hydrate and perfused with ice-cold PBS followed by $4 \%$ paraformaldehyde. Brains were removed and cryoprotected by immersion in PBS with $10-30 \%$ sucrose. Thirty micrometer sections were cut in the parasagittal or coronal planes using a sliding microtome and floated in $500 \mu \mathrm{l}$ of blocking solution (PBS with 10\% normal goat serum) for $1 \mathrm{hr}$. Sections were permeabilized with $0.1 \%$ Triton X-100 in blocking solution for $1 \mathrm{hr}$, washed with blocking solution, and incubated overnight with either affinity-purified Srg1 antiserum (1:100-1:150 in blocking solution) or an equal concentration (0.13 $\mu \mathrm{g} / \mathrm{ml}$ ) of rabbit $\mathrm{IgG}$ as a negative control. Sections were washed with blocking solution three times. For immunofluorescence, sections were incubated with Cy3-conjugated anti-rabbit antisera (1:3000; Jackson ImmunoResearch, West Grove, PA) for $1 \mathrm{hr}$. Sections were washed with PBS and subsequently with PBS with $1 \mu \mathrm{g} / \mathrm{ml} \mathrm{4}$ ',6-diaminido-2-phenylindole (DAPI) and were then mounted on glass slides using VectaShield mounting medium (Vector Laboratories, Burlingame, CA). For immunohistochemistry, sections were incubated with horseradish peroxidase-conjugated secondary antiserum using the Vectastain Elite Kit according to the specifications of the manufacturer. 3,3'-Diaminobenzidine (DAB) was used as the peroxidase substrate. Sections were mounted on glass slides, dehydrated, and mounted with DPX mountant (Fluka, Buchs, Switzerland). Digital images were obtained using a Zeiss Axiocam with a Zeiss Axiophot microscope (Zeiss, Thornwood, NY). Images were processed using Adobe Photoshop (version 5.5) software (Adobe Systems Inc., San Jose, CA).

In situ hybridization. Thirty micrometer sections were prepared from P15 rat brains as described previously (Thompson, 1996). The probe for Srg1 spanned nt 783-1574 (Thompson, 1996). The probe for calbindin was generated by PCR amplification of the full-length cDNA from a rat brain cDNA library using gene-specific primers. The calbindin cDNA was subcloned into plasmid pCR2.1 (Invitrogen, San Diego, CA) in both orientations. Sense and antisense digoxigenin-labeled cRNA probes were generated by in vitro transcription according to the specifications of the manufacturer (Roche, Welwyn Garden City, UK). Probes were hydrolyzed to an average size of $150 \mathrm{bp}$ and used for in situ hybridization as described previously (Giger et al., 1998), except that overnight hybridization was at $65^{\circ} \mathrm{C}$ and incubation with substrate was for $48 \mathrm{hr}$.

\section{RESULTS}

\section{Srg1 is expressed only in brain}

The tissue distribution of Srg1 mRNA and protein was analyzed using Northern and Western blot analyses. To determine whether Srg1 RNA expression is brain-specific, we used Northern blot analysis of RNA isolated from various adult rat tissues (Fig. 1). Srg1 mRNA was detected in the brain but not in the other tissues tested. Within the brain, Srg1 is present at higher levels in the cerebellum relative to the forebrain.

To examine the expression of Srg1 protein, a polyclonal antiserum was raised to a bacterially expressed fusion protein comprising the C-terminal 213 aa of Srg1 (Fig. 2A). In cerebellar protein extracts, this antiserum recognized a single band of $\sim 45$ $\mathrm{kDa}$, the predicted size of the Srg1 protein (Fig. $2 B$ ). Detection of a single band suggests that this antiserum does not cross-react with other synaptotagmins. Specificity was confirmed by performing Western blot analysis in the presence of specific and nonspe- 

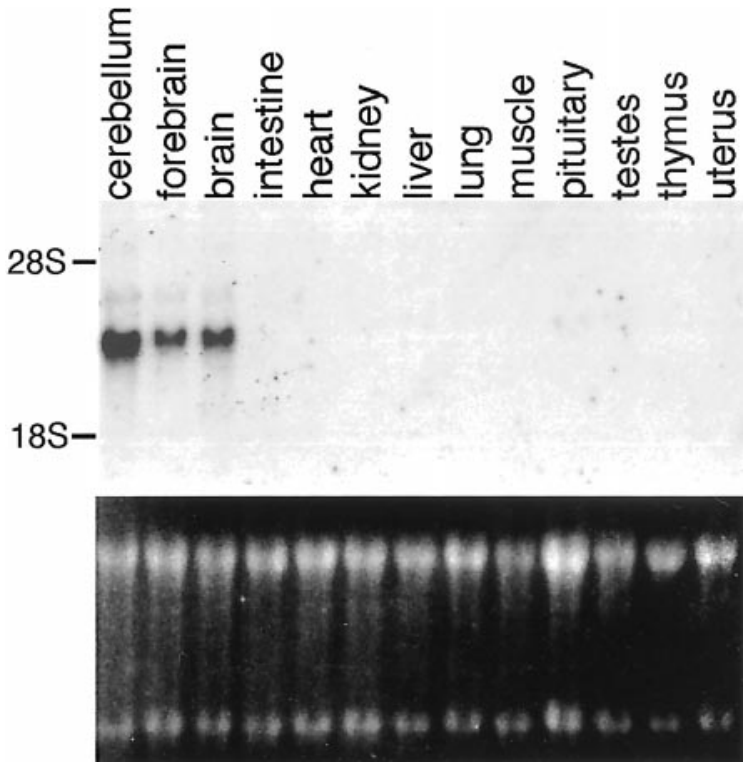

Figure 1. Srg1 RNA is brain-specific. A Northern blot analysis of total RNA $(15 \mu \mathrm{g} /$ lane $)$ obtained from various rat tissues using a ${ }^{32} \mathrm{P}$-labeled Srg1-specific cDNA probe is shown. Top panel, Autoradiograph of Northern blot. Bottom panel, Ethidium bromide-stained gel to verify RNA loading. The relative positions of $28 \mathrm{~S}$ and $18 \mathrm{~S}$ RNAs are indicated.

cific competitor proteins. The signal for Srg1 was abolished in the presence of excess Srg1 fusion protein but not by bacterial protein (GST) or bacterially expressed SytI, indicating that the antiserum is specific for Srg1. Using this Srg1-specific antiserum, we examined the expression of Srg1 in protein extracts prepared from adult rat tissues by Western blot analysis (Fig. 2C). In agreement with our results for Srg1 RNA, Srg1 protein was detected in the brain and not in various other tissues. Thus, Srg1 is expressed at detectable levels only in the nervous system.

\section{Hypothyroidism causes reduced Srg1 expression}

Because Srg1 expression in the brain is both developmentally regulated and regulated by $\mathrm{TH}$, we examined the effect of $\mathrm{TH}$ on Srg1 expression in the developing brain. RNA was isolated from whole brains at various developmental ages from birth (P0) to P55 from both hypothyroid and euthyroid animals and used for Northern blot analysis (Fig. 3A). In euthyroid (normal) animals, low levels of Srg1 RNA were detected at birth (P0) and increased markedly during the first three postnatal weeks (P0-P20). In hypothyroid animals, Srg1 expression was also detected early in postnatal development (P0-P2), but expression after P7 was significantly lower than in euthyroid animals. Thus, TH deficiency results in decreased expression of Srg1 RNA at specific developmental ages, which correlate with the developmental time period (P5-P20) within which $\mathrm{TH}$ is known to influence neural development.

Having determined the developmental profile of Srg1 RNA expression in whole brain, we subsequently examined the effect of TH deficiency on Srg1 protein levels. To examine Srg1 protein levels during postnatal brain development, we analyzed Srg1 protein expression in various brain regions. The cerebellum, somatosensory cortex, and hippocampus were chosen for study because these regions are affected in the hypothyroid brain. Protein extracts prepared from the indicated brain regions from euthyroid and hypothyroid pups were used for Western blot analysis (Fig. 3B). TH deficiency causes a decrease in Srg1 pro-
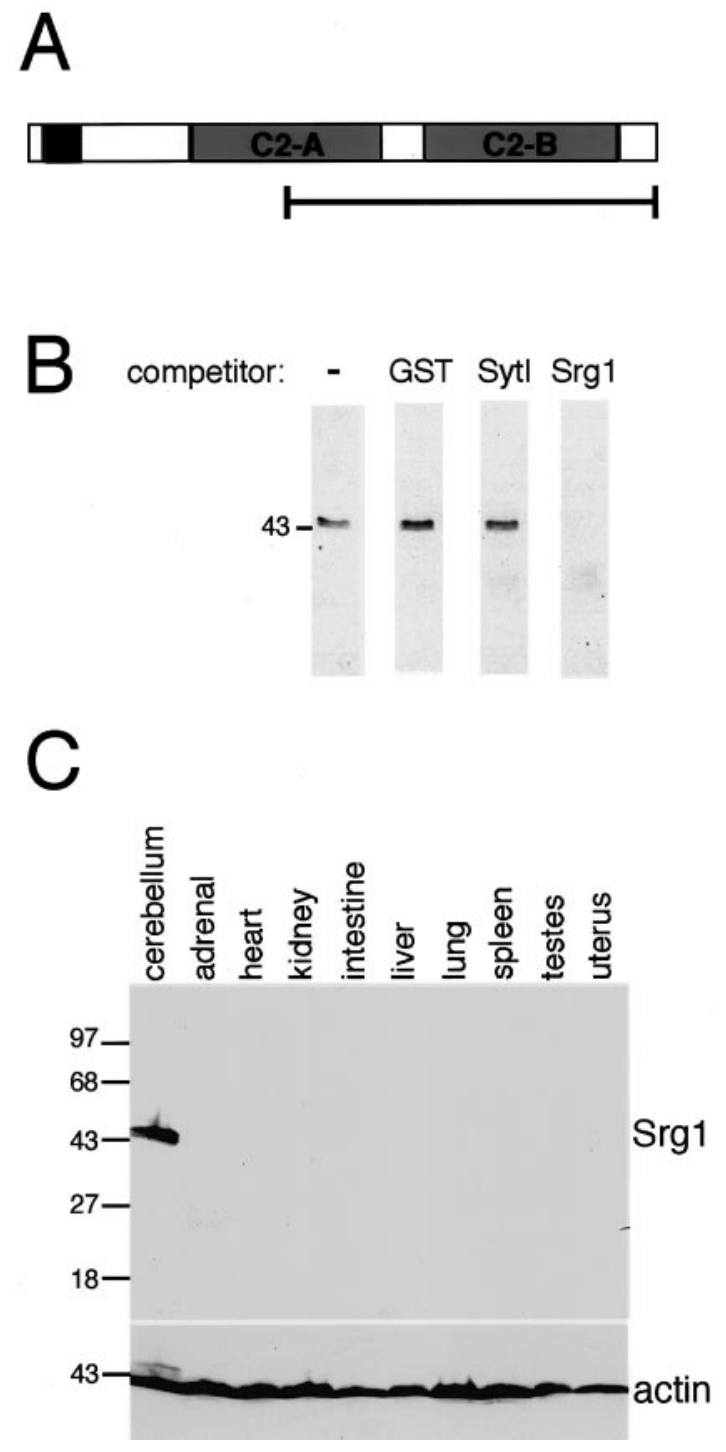

Figure 2. Srg1-specific antiserum recognizes a $45 \mathrm{kDa}$ protein only in rat brain. $A$, Schematic representation of Srg1 protein structure. The bracketed line indicates the part of the Srg1 protein produced in bacteria and used for generating Srg1-specific antiserum. $B$, Western blot analysis of protein extract from a P15 rat cerebellum. Affinity-purified Srg1-specific antiserum recognizes a single $45 \mathrm{kDa}$ protein. Detection of this band is abolished in the presence of excess bacterially expressed Srg1 fusion protein $(\operatorname{Srg} 1)$ but is unaffected by excess nonspecific bacterial protein (GST) or bacterially expressed SytI fusion protein. $C$, Western blot analysis of protein extracts from various rat tissues using Srg1-specific antiserum. Top panel, Western blot with Srg1-specific antiserum visualized by ECL. Bottom panel, Same blot probed with $\alpha$-actin-specific antiserum to verify protein loading. Molecular masses (in kilodaltons) are indicated on the left.

tein levels in all regions. In the euthyroid cerebellum and cortex, Srg1 protein is first detected at P10, whereas in hypothyroid animals, Srg1 protein is not detected in these brain regions until P20. Despite this lag in expression, Srg1 protein reaches nearly normal levels in hypothyroid adult animals. In the hippocampus, Srg1 protein is detected at P10 in both euthyroid and hypothyroid animals, and Srg1 abundance differs between euthyroid and hypothyroid animals only at P25 and P60. Thus, Srg1 expression is less sensitive to TH levels in the hippocampus than in other brain regions. Together these results indicate that $\mathrm{TH}$ deficiency causes 

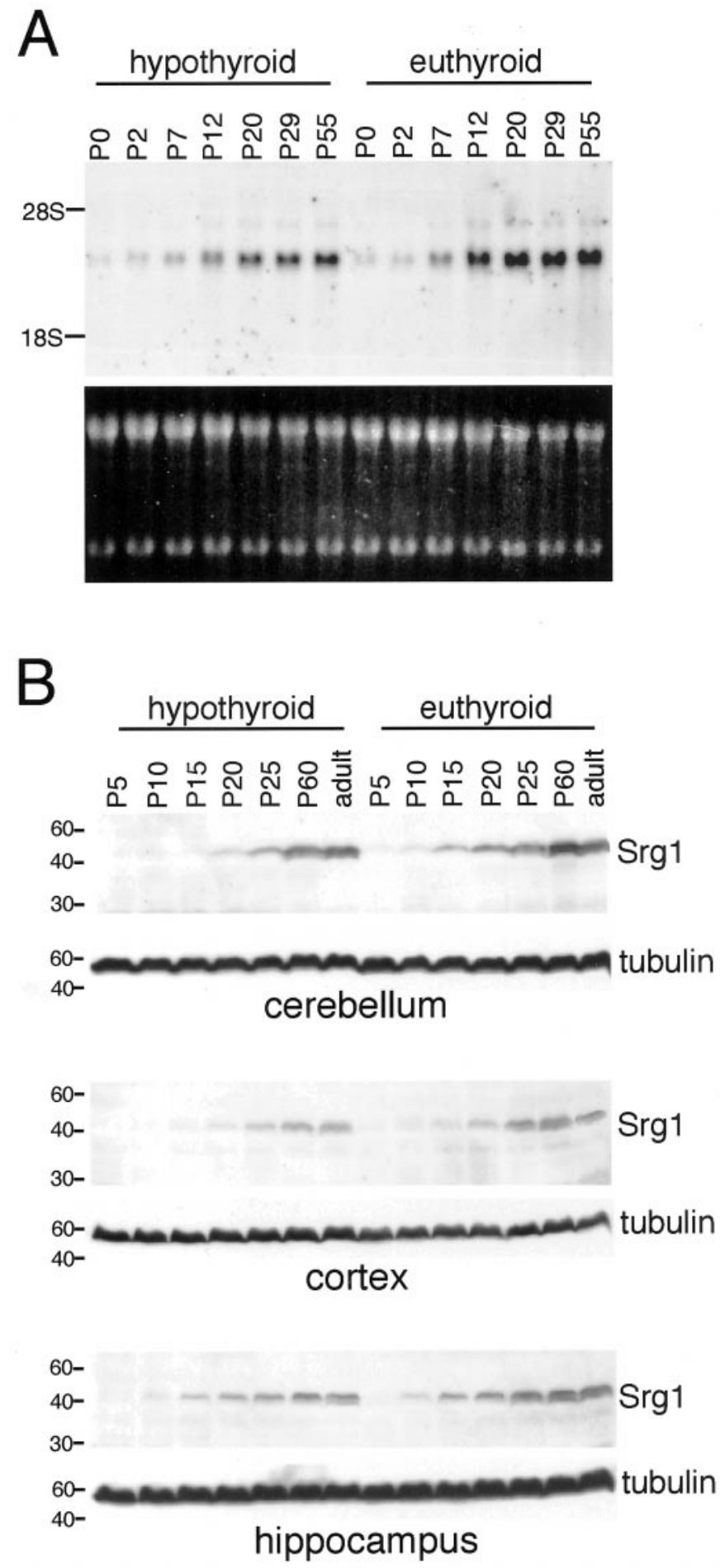

Figure 3. Effect of TH on Srg1 mRNA and protein expression during development. RNA and protein were isolated from normal (euthyroid) and methimazole-treated (hypothyroid) rats at different stages of postnatal development (P0 to adult) and used for Northern and Western blot analyses. $A$, Northern blot analysis of total RNA ( $15 \mu \mathrm{g} /$ lane) from whole brain using a ${ }^{32} \mathrm{P}$-labeled Srg1 cDNA probe. Top panel, Autoradiograph of Northern blot. Bottom panel, Ethidium bromide-stained gel. B, Western blot analysis of protein extracts prepared from the cerebellum, somatosensory cortex (cortex), and hippocampus. Top of each panel, Western blot analysis using Srg1-specific antiserum. Bottom of each panel, Same blot probed with $\beta$-tubulin antiserum to verify protein loading. Molecular masses (in kilodaltons) are indicated on the left. a reduction in $\operatorname{Srg} 1$ protein expression during postnatal brain development and that the degree of reduction is dependent on the brain region and developmental age examined.

\section{In vivo expression of Srg1 protein}

To assess the in vivo distribution of Srg1 protein, we examined Srg1 expression using immunohistochemistry. The cytoarchitecture of the developing cerebellum consists of internal granule cell layers (IGLs) and Purkinje cell layers, a neurite and synapse-rich molecular layer (ML), and an external granule cell layer (EGL) composed of actively dividing cells (Fig. 4A). Previous in situ hybridization results showed that $\mathrm{Srg} 1$ is highly expressed in the IGL but absent in the EGL (Thompson, 1996).

Distribution of Srg1 in vivo was analyzed using sections from neonatal rat cerebellum (P16). Using Srg1-specific antiserum, we detected Srg1 immunoreactivity in the IGL and pronounced expression in the ML. As expected, no Srg1 protein was detected in the EGL. Specificity of the antibody staining was confirmed by competition with specific and nonspecific proteins (Fig. 4B). The ML is densely packed with axons projecting from granule cells (parallel fibers) and the dendritic trees of Purkinje cells. Detection of Srg1 protein in the ML together with previous in situ hybridization results showing high levels of Srg1 mRNA in granule cells (Thompson, 1996) suggested that the Srg1 protein present in the molecular layer is localized to granule cell axons. To confirm that the Srg1 in the ML is primarily from granule cells and not Purkinje cells, we used in situ hybridization to detect expression of Srg1 RNA and a Purkinje cell-specific RNA (calbindin) (Fig. 4B). As expected, Srg1 RNA is detected in cells in the IGL and not in Purkinje cell bodies, whereas under the same conditions calbindin RNA is clearly detected in Purkinje cells.

Because TH is known to influence the development of brain regions in addition to the cerebellum, the expression of Srg1 protein was also examined in other brain regions (Fig. 5). Srg1specific antiserum was used to detect Srg1 protein in a series of coronal sections from neonatal (P12) rat brain. High levels of Srg1 were detected in several regions of the brain, including the cortex, hippocampus, globus pallidus, habenula, and multiple thalamic nuclei. Interestingly, previous in situ hybridization results showed Srg1 RNA expression in the striatum (caudate putamen) (Thompson, 1996), whereas expression of Srg1 protein in the striatum is relatively low. However, because cells from the striatum send projections to the globus pallidus, Srg1 protein detected in the globus pallidus likely originates from cells in the striatum. Thus, as in the cerebellum, $\mathrm{Srg} 1$ protein appears to localize to neuronal processes.

\section{Srg1 expression in cultured cerebellar granule cells}

Because analyzing expression in whole brain or particular brain regions is limited in assessing the relative contributions of different cell types, we sought to investigate Srg1 expression in a defined population of cells. Because Srg1 is highly expressed in the granule cells of the cerebellum, we used dissociated primary cultures of CGCs. In culture, CGCs undergo a program of differentiation that includes neurite extension, establishment of neuronal polarity, and synapse formation. After 5-6 d in vitro (DIV), CGCs establish a dense neuritic network with numerous synapses and are functionally and morphologically differentiated by 7-9 DIV (Gallo et al., 1982; Kingsbury et al., 1985). To examine the expression of Srg1 protein during this period of neuronal development in vitro, we prepared whole-cell lysates from CGCs that had been cultured for 1-9 DIV. Total protein 

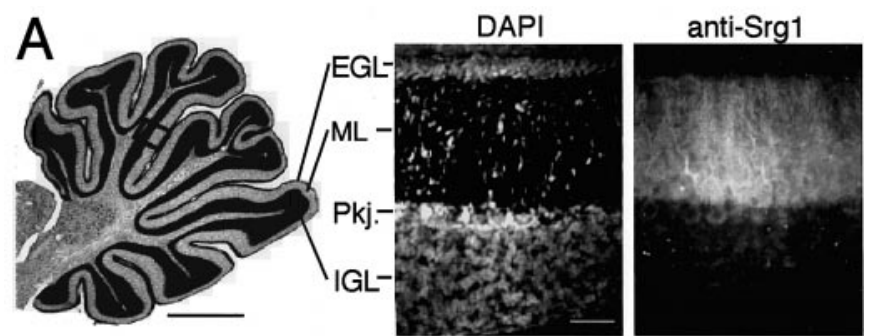

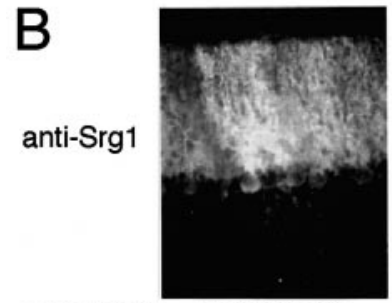

competitor: nonspecific

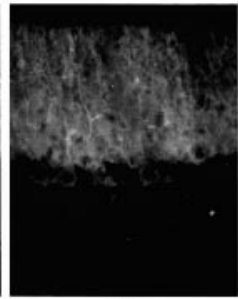

Sytl

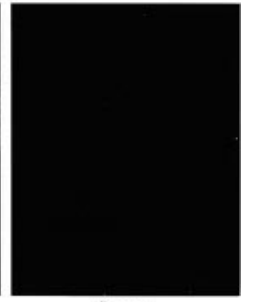

Srg1
C

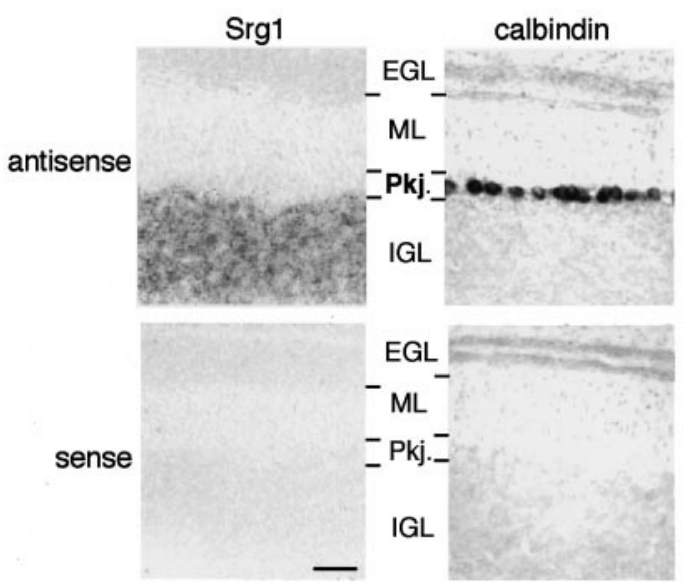

Figure 4. Expression of Srg1 in the neonatal cerebellum. A, Immunofluorescent staining of a parasagittal section from a P16 rat cerebellum. Left, Low-magnification (scale bar, $1 \mathrm{~mm}$ ) photomicrograph of a parasagittal section of a P16 rat cerebellum stained with cresyl violet. Positions of the EGL, ML, Purkinje cell layer $(P k j$.$) , and IGL are indicated. The$ box indicates the region shown at high magnification (scale bar, $50 \mu \mathrm{m}$ ) to the right. Middle, DAPI staining to mark cell nuclei. Right, Immunofluorescence image of Srg1 protein detected using Srg1-specific antiserum and Cy3-coupled anti-rabbit antiserum. Srg1 immunoreactivity is prominent in the molecular layer. $B$, Competition experiment demonstrating the specificity of Srg1 antiserum. Signal is abolished in the presence of excess bacterially expressed $\operatorname{Srg} 1$ fusion protein $(\operatorname{Srg} 1)$ but not in the presence of nonspecific bacterial proteins (nonspecific) or a synaptotagmin I fusion protein (SytI). C, In situ hybridization showing Srg1 RNA specifically in the IGL. Coronal sections from P15 rat brain were hybridized with antisense strand (top panels) or sense strand (bottom panels) cRNA probes for Srg1 (left panels) or calbindin (right panels). Positions of the EGL, ML, Purkinje cell layer $(P k j$.), and IGL are indicated. Scale bar, $50 \mu \mathrm{m}$.

from each lysate was analyzed by Western blot analysis to determine the levels of Srg1 (Fig. 6A). Srg1 protein becomes detectable at 3 DIV, rises dramatically at 6 DIV, and plateaus at 7 DIV. Thus, expression of Srg1 is correlated with the mature, differentiated state of these cells in vitro. This is consistent with our in vivo results, which showed that Srg1 is expressed in mature granule cells in the neonatal cerebellum. This suggests that the effects on Srg1 observed in vivo occur primarily because of expression in granule cells and also indicates that cultured CGCs are an appropriate in vitro model.

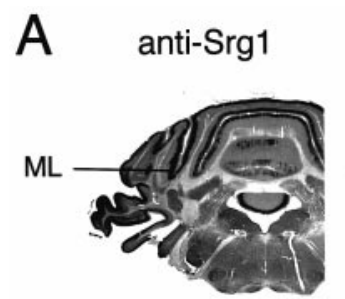

Control
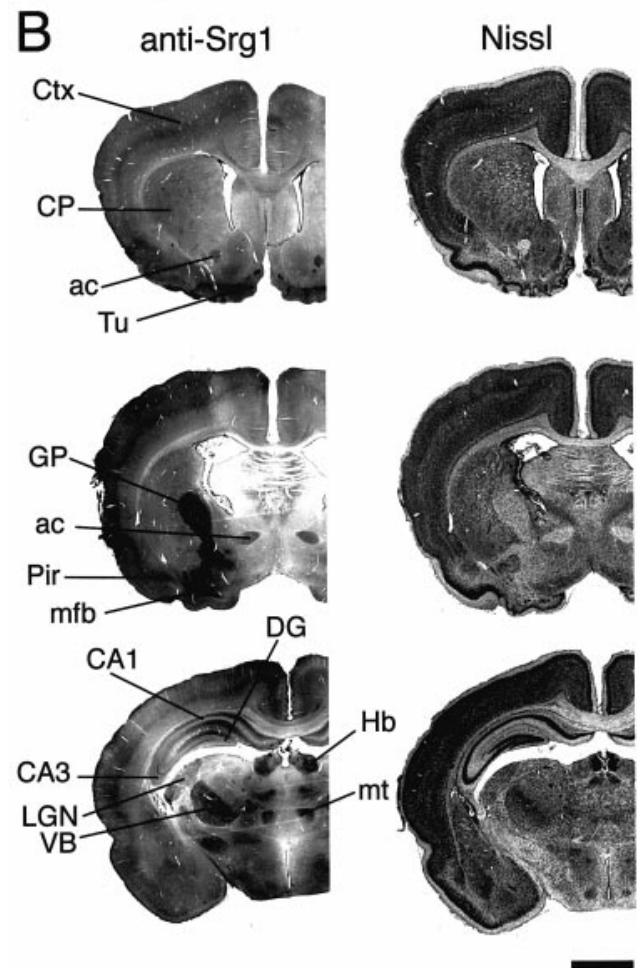

Figure 5. Srg1 is expressed in multiple brain regions. $A$, Srg1-specific antiserum (anti-Srg1) and rabbit IgG (Control) were incubated with coronal sections from a P12 rat cerebellum. Detection was with peroxidasecoupled secondary antiserum using DAB as the substrate. Staining in the ML is indicated. $B$, Expression of Srg1 in the neonatal rat brain. Staining with Srg1-specific antiserum (anti-Srg1) in coronal sections from a P12 rat brain is shown. Sections are shown rostral (top) to caudal (bottom). Nissl, Cresyl violet staining; $a c$, anterior commissure; $C A 1, C A 3$, regions of hippocampus; $C P$, caudate putamen (striatum); $C t x$, cortex; $D G$, dentate gyrus; $G P$, globus pallidus; $H b$, habenula; $L G N$, lateral geniculate nucleus of the thalamus; $m f b$, medial forebrain bundle; $m t$, mamillothalamic tract; Pir, piriform cortex; $T u$, olfactory tubercle; $V B$, ventrobasal nuclear complex of the thalamus. Scale bar, $2 \mathrm{~mm}$.

\section{Srg1 is regulated by thyroid hormone in neurons}

Having established that Srg1 is expressed in cultured CGCs, we subsequently tested whether Srg1 expression is regulated by $\mathrm{TH}$ in this in vitro model. CGCs were grown in the absence of $\mathrm{TH}$ and then treated with TH for $24 \mathrm{hr}$. We observed no gross morphological differences between normal and TH-deficient cultures and no significant difference in cell survival (data not shown). RNA was prepared and used for Northern blot analysis (Fig. 6B). As we observed previously in intact animals, Srg1 expression was reduced in the absence of TH. To investigate the kinetics of Srg1 expression in cultured CGCs, cells were treated with $\mathrm{TH}$ for various periods of time and then harvested for Northern blot analysis (Fig. 6C). Levels of Srg1 RNA increased two-fold within $4 \mathrm{hr}$, reaching a 

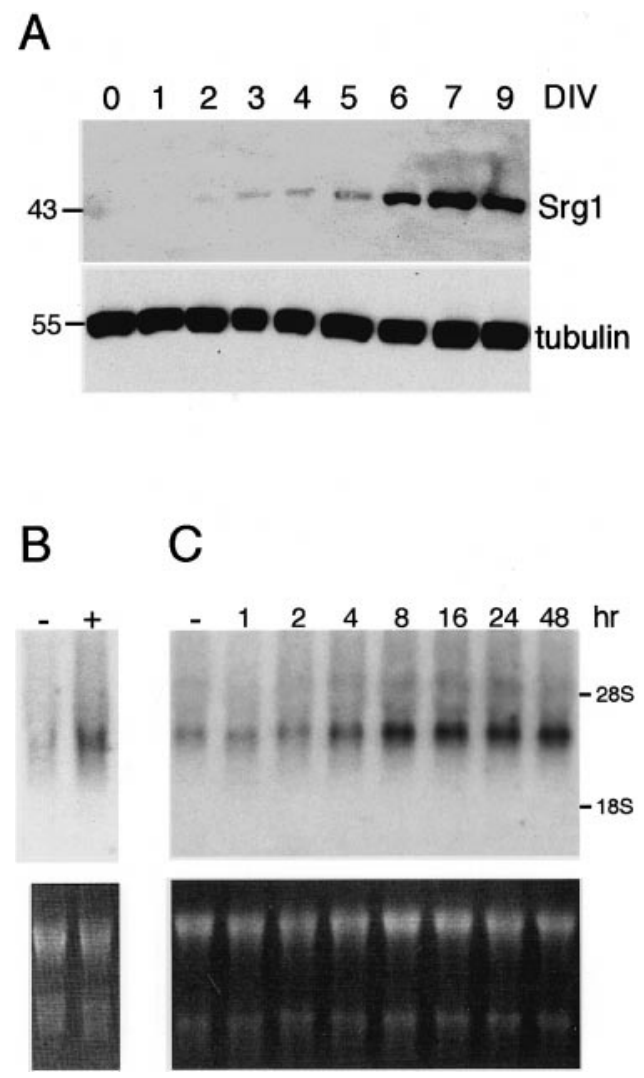

Figure 6. Expression of Srg1 in cultured cerebellar neurons. A, Protein extracts were prepared from cultured CGCs at various times after plating (1-9 d) and used for Western blot analysis. Top panel, Western blot analysis using Srg1-specific antiserum. Bottom panel, Same blot probed with $\beta$-tubulin antiserum to verify protein loading. Molecular masses (in kilodaltons) are indicated. $B$, Northern blot analysis of RNA from CGCs grown in the absence $(-)$ or presence $(+)$ of TH. Top panel, Autoradiograph of Northern blot. Bottom panel, Ethidium bromide-stained gel. $C$, Srg1 is rapidly induced by TH in CGCs. CGCs were grown in the absence of TH and then treated with TH for various periods of time. A Northern blot analysis (10 $\mu \mathrm{g}$ total RNA per lane) using a ${ }^{32} \mathrm{P}$-labeled Srg1-specific cDNA probe is shown. Top panel, Autoradiograph of Northern blot. Bottom panel, Ethidium bromide-stained gel. $h r$, Hours of TH treatment.

plateau (3.5-fold) at $8 \mathrm{hr}$. This rapid induction of Srg1 expression suggests that regulation by $\mathrm{TH}$ is direct and not a secondary effect of $\mathrm{TH}$ treatment. We also analyzed Srg1 protein expression in cultured CGCs. Protein extracts were prepared from CGCs grown in the absence and presence of $\mathrm{TH}$ and used for Western blot analysis (Fig. 7). Similar to the RNA levels, Srg1 protein levels were significantly lower in CGCs cultured in the absence of TH.

Although we have shown that Srg1 is highly expressed in cerebellar granule cells, the cerebellum is also populated by other cell types, such as glial cells. To determine whether Srg1 expression is specific to neurons and therefore may have a function specific to neurons, Srg1 expression was also examined in glial cells. Protein extracts were prepared from primary cultures of glial cells and used for Western blot analysis (Fig. 7). Srg1 protein was not detected in cultured astroglial cells from either the cerebral cortex (Fig. 7) or the cerebellum (data not shown), whereas a glial marker (GFAP) was detected. In addition, Srg1 protein was not detected in an oligodendrocytic cell line (Louis et al., 1992) (data not shown). Thus, among the cell types examined Srg1 is selectively expressed in neurons.

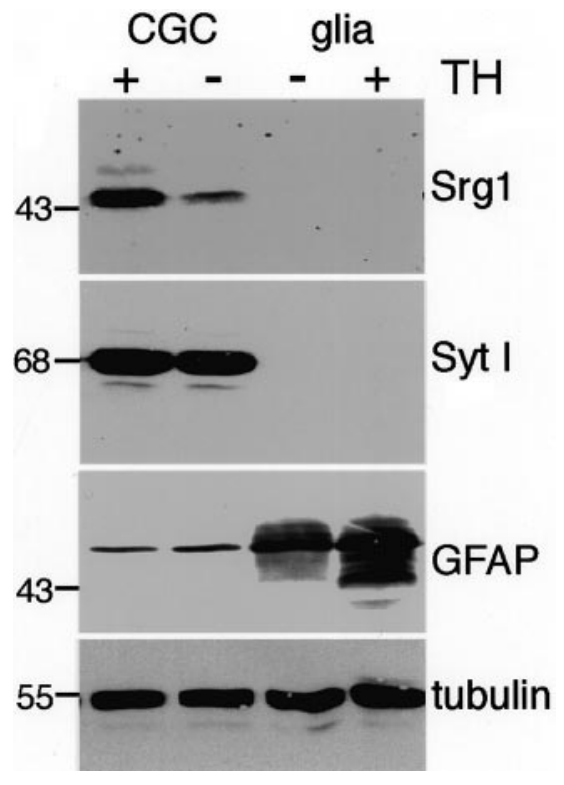

Figure 7. Srg1 is specifically expressed in neurons. A Western blot analysis of protein extracts from cultured CGCs and glial cells grown in the absence $(-)$ and presence $(+)$ of $\mathrm{TH}$ is shown. The same blot was sequentially probed with polyclonal anti-Srg1, monoclonal anti-SytI, antiGFAP, and anti- $\beta$-tubulin antibodies. Positions of molecular mass markers (in kilodaltons) are indicated.

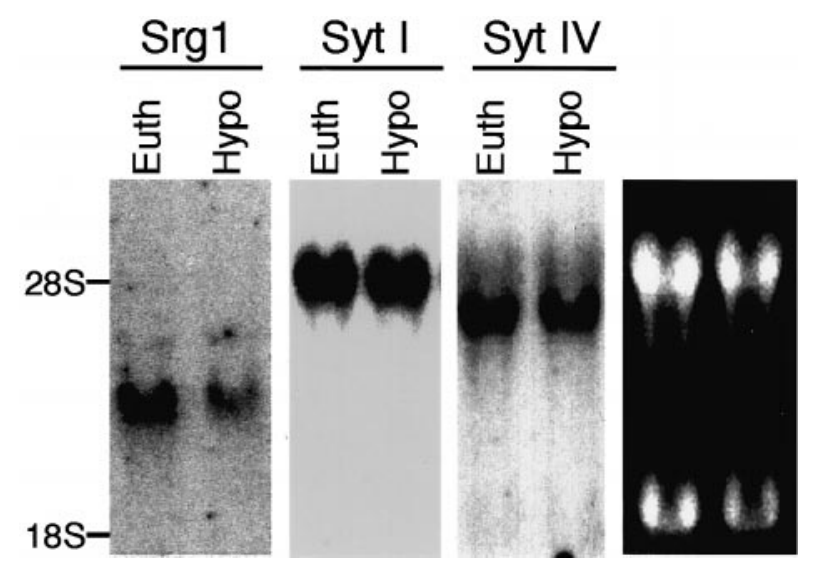

Figure 8. Expression of synaptotagmins I and IV is unaffected in hypothyroid animals. Total RNA (15 $\mu \mathrm{g} /$ lane) prepared from cerebella of hypothyroid (Hypo) or euthyroid (Euth) P16 rats was used for Northern blot analysis with radiolabeled Srg1, SytI, and SytIV cDNA probes. Right panel, Ethidium bromide-stained gel shows equivalent loading of RNA.

\section{Expression of other synaptotagmins is not regulated by $\mathrm{TH}$}

Because Srg1 is related to a multigene family, we assessed whether TH-regulated expression was a property of other synaptotagmin genes. Synaptotagmin I protein levels were not affected by TH status in cultured CGCs, indicating that the effect on Srg1 expression is specific (Fig. 7). We also tested whether a synaptotagmin whose expression is induced by depolarization (synaptotagmin IV; Vician et al., 1995) could also be induced by TH. Expression of synaptotagmins I and IV in hypothyroid and euthyroid neonatal (P16) rat brains were analyzed using Northern blot analysis (Fig. 8). The levels of synaptotagmin I and IV mRNA did not differ in the hypothyroid and euthyroid brains, indicating that regulation by TH may be a unique property of Srg1. 


\section{DISCUSSION}

Differential gene expression is one of the mechanisms guiding mammalian brain development. $\mathrm{TH}$, by binding to nuclear receptors that are transcription factors, exerts specific effects on brain development by regulating gene expression (Oppenheimer and Schwartz, 1997; Zhang and Lazar, 2000). Here we report the temporal and spatial expression of a recently identified $\mathrm{TH}$ responsive gene, Srg1, during postnatal rat brain development. We find that Srg1 RNA and protein are expressed specifically in the nervous system and that expression is correlated with brain regions and structures influenced by $\mathrm{TH}$.

\section{Srg1 expression in cerebellar development}

The cytoarchitecture of the developing cerebellum consists of internal granule cell and Purkinje cell layers, a neurite- and synapse-rich ML, and an EGL composed of actively dividing cells (Fig. 4A). Cerebellar development occurs in a temporally precise manner, beginning postnatally (Altman, 1972a,b). Between P7 and $\mathrm{P} 10$, the EGL is at its maximum size. As proliferating neuroblasts in the EGL undergo terminal mitosis, becoming basket, stellate, or granule cells, these cells migrate inward toward their final destination, the IGL. Consequently, the width of the EGL decreases to approximately three to four cells by P16-P19 and disappears by P24 as differentiated cells fully populate the IGL. As granule cells differentiate and migrate, their axons grow first in a horizontal plane and then vertically as the cells migrate through the Purkinje cell layer. Thus, the bulk of the ML is composed of parallel fibers projecting from granule cells in the IGL, and the width of the ML increases as the size of the EGL decreases. Parallel fibers eventually form mature synapses on Purkinje cell dendrites starting at approximately P15 (Altman, 1972b).

By examining Srg1 expression at different developmental ages, we detect Srg1 protein beginning at P10, a stage when a significant number of postmitotic granule neurons accumulate in the IGL. The timing of Srg1 protein expression, together with previous results showing that Srg1 is present in the IGL but not in the EGL (Thompson, 1996), suggests that Srg1 protein is expressed primarily by postmitotic neurons in the cerebellar cortex. Consistent with these results, our immunohistochemical analysis showed that Srg1 protein is present in the IGL and absent in the EGL.

\section{TH deficiency and Srg1 expression}

Neonatal TH deficiency markedly affects Srg1 expression in the developing cerebellum. Srg1 protein expression in the hypothyroid cerebellum is delayed, as Srg1 is first detected at P20 rather than at P10. This delay in Srg1 expression is correlated with the characteristic delay in maturation and migration of EGL cells observed in the hypothyroid cerebellum (Nicholson and Altman, 1972a; Rabie and Legrand, 1973). Although the EGL disappears by P24 in the euthyroid cerebellum, the EGL persists significantly longer in the hypothyroid cerebellum (up to P30) because of delayed cell migration and/or differentiation (Nicholson and Altman, 1972a; Rabie and Legrand, 1973). This suggests that Srg1 plays a role in granule cell differentiation. Alternatively, the delay in granule cell differentiation and migration may be responsible for altered Srg1 expression, because there may simply be fewer mature cells at this developmental stage. However, our in vitro experiments using isolated cerebellar granule cells in culture support the idea that $\mathrm{TH}$ acts directly on granule cells. For example, $\operatorname{Srg} 1 \mathrm{mRNA}$ levels increase rapidly after exposure to $\mathrm{TH}$, and the regulation and expression of Srg1 in cultured CGCs parallel in vivo results.
Although Srg1 protein expression is reduced by $\mathrm{TH}$ deficiency during the second and third postnatal weeks, Srg1 protein levels are nearly normal in the hypothyroid adult cerebellum. This pattern of regulation is similar to other TH-regulated genes such as myelin basic protein (MBP) (Strait et al., 1992). More importantly, in this respect $\mathrm{Srg} 1$ expression parallels the hypothyroid phenotype. Despite the delay in granule cell migration, the EGL eventually disappears, and the overall organization of the cerebellar cortex appears normal in the hypothyroid cerebellum. However, the altered cerebellar maturation caused by $\mathrm{TH}$ deficiency is functionally significant, because the delay in migration of cells from the EGL is thought to underlie the observed decrease in the number and density of synaptic contacts between granule cells and Purkinje cells (Nicholson and Altman, 1972b; Legrand, 1979).

\section{Srg1 expression in the cerebellar molecular layer}

Immunohistochemical detection of Srg1 protein in the neonatal cerebellum showed that Srg1 protein is most abundant in the ML, the region into which granule cells project axons and establish synaptic contacts with Purkinje cells. The finding that Srg1 is abundant in the ML suggests that Srg1 protein accumulates primarily in granule cell axons. Srg1 immunoreactivity is also coincident with Purkinje cell bodies. Localization of Srg1 protein in parallel fibers could explain this appearance, because light and electron microscopic analyses have shown that parallel fibers are contiguous with Purkinje cell dendrites and secondary branches at this stage of development (Altman, 1972b). Results of the in situ hybridization analysis confirmed that Purkinje cells do not express Srg1 RNA. The appearance of Srg1 protein on Purkinje cells is also consistent with Srg1 expression by basket cells, which form synapses on Purkinje cell bodies starting at approximately P10. Because these cells comprise a small proportion of cells $(<10 \%)$ in the cerebellar cortex, it is unlikely that they contribute the majority of Srg1 immunoreactivity. Similarly, evidence that Srg1 is not expressed by cultured cerebellar glial cells indicates that this is not a primary source of Srg1 immunoreactivity. Analysis of Srg1 expression in other brain regions is consistent with localization to neuronal processes. For example, Srg1 protein present in the globus pallidus likely arises from axonal projections from the striatum.

The localization of Srg1 protein to neurites, together with structural similarity to synaptotagmins, implicates Srg1 in synapse formation and/or function. During development, Srg1 expression precedes the formation of mature synapses between parallel fibers and Purkinje cell dendrites. Notably, synapse formation between parallel fibers and Purkinje cells is reduced in the TH-deficient brain (Nicholson and Altman, 1972a,b). Our preliminary experiments suggest that Srg1 does not colocalize with synaptic vesicle markers and thus may have a function distinct from other synaptotagmins. Consistent with this idea is evidence that expression of other synaptotagmins is not influenced by $\mathrm{TH}$.

\section{TH and the developing brain}

In addition to effects on cerebellar development, TH deficiency causes alterations throughout the brain, including decreased myelination, decreased synaptogenesis, and altered morphology of multiple cell types. By analyzing other brain regions, we found that Srg1 expression initiates postnatally in the somatosensory cortex and hippocampus within the critical period of TH action in the brain. TH deficiency results in decreased Srg1 expression in both regions, although to a lesser degree in the hippocampus. This difference in TH-sensitive hippocampal expression may explain why changes in Srg1 expression detected in whole brain are 
not as striking as in the isolated cerebellum. Decreased Srg1 expression in these regions is correlated with TH-dependent developmental defects. Specific alterations in dendritic morphology have been identified in pyramidal cells in the cerebral cortex and in granule and pyramidal cells in the hippocampus (Schwartz, 1983; Rami et al., 1986). In the hypothyroid somatosensory cortex, cells are smaller and more closely aggregated than normal, in part because of decreased development of axonal and dendritic processes (Schwartz, 1983).

\section{TH-responsive gene expression in developing cerebellum}

Few genes regulated by $\mathrm{TH}$ in the brain have been identified (Oppenheimer and Schwartz, 1997; Thompson and Potter, 2000). In addition to $\mathrm{Srg} 1$, genes that are regulated by $\mathrm{TH}$ in the cerebellum include MBP, calbindin, Purkinje cell protein-2 (Pcp2), and hairless (hr) (Farsetti et al., 1991; Strait et al., 1992; Thompson, 1996). MBP is expressed by oligodendrocytes and functions in myelination. Decreased expression of MBP in hypothyroid animals may partially explain the phenotype of decreased myelination observed in the hypothyroid brain (Schwartz, 1983). In the cerebellum, both calbindin and Pcp-2 are expressed specifically in Purkinje cells (Nordquist et al., 1988). Calbindin is a calcium-binding protein proposed to have a buffering function that protects cells against increases in intracellular calcium (Baimbridge et al., 1992). The function of Pcp-2 is not known, although Purkinje cell-specific expression of both proteins suggests that they could be involved in mediating the effects of TH on Purkinje cells. We have shown recently that expression of the $h r$ gene is directly regulated by $\mathrm{TH}$ in cerebellar granule cells (Thompson and Potter, 2000). The protein encoded by $h r(\mathrm{Hr})$ functions as a transcriptional corepressor for $\mathrm{TH}$ receptors, suggesting that $\mathrm{Hr}$ influences the expression of other TH-responsive genes and thus may be a central mediator of TH action (Thompson and Bottcher, 1997; Thompson and Potter, 2000).

Like these genes, $\mathrm{Srg} 1$ is part of a program of gene expression induced by TH in the developing brain. Srg1 is likely an important component of this program, because Srg1 expression is developmentally regulated and specific to neurons. Furthermore, Srg1 expression is coincident with cell types and structures affected by $\mathrm{TH}$ deficiency. Although it is likely that $\mathrm{TH}$ regulates the expression of many genes during neural development, the temporal and spatial pattern of Srg1 expression supports a putative role for Srg1 in mediating TH-dependent developmental processes.

\section{REFERENCES}

Altman J (1972a) Postnatal development of the cerebellar cortex in the rat. I. The external germinal layer and the transitional molecular layer. J Comp Neurol 145:353-398.

Altman J (1972b) Postnatal development of the cerebellar cortex in the rat. II. Phases in maturation of Purkinje cells and of the molecular layer. J Comp Neurol 145:399-464.

Baimbridge KG, Celio MR, Rogers JH (1992) Calcium-binding proteins in the nervous system. Trends Neurosci 15:303-308.

Bajjalieh SM, Scheller RH (1995) The biochemistry of neurotransmitter secretion. J Biol Chem 270:1971-1974.

Chomczynski P, Sacchi N (1987) Single-step method of RNA isolation by acid guanidinium thiocyanate-phenol-chloroform extraction. Anal Biochem 162:156-159.

Eayrs JT (1971) Thyroid and developing brain: anatomical and behavioral effects. In: Hormones in development (Hamburgh M, Barrington EJW, eds), pp 345-355. New York: Meredith.

Facchinetti F, Hack NJ, Balazs R (1998) Calcium influx via ionotropic glutamate receptors causes long-lasting inhibition of metabotropic glutamate receptor-coupled phosphoinositide hydrolysis. Neurochem Int $33: 263-270$.

Farsetti A, Mitsuhashi T, Desvergne B, Robbins J, Nikodem VM (1991)
Molecular basis of thyroid hormone regulation of myelin basic gene expression in rodent brain. J Biol Chem 266:23226-23232.

Gallo V, Ciotti MT, Coletti A, Aloisi F, Levi G (1982) Selective release of glutamate from cerebellar granule cells differentiating in culture. Proc Natl Acad Sci USA 79:7919-7923.

Geppert M, Goda Y, Hammer RE, Li C, Rosahl TW, Stevens CF, Südhof TC (1994) Synaptotagmin I: a major $\mathrm{Ca}^{2+}$ sensor for transmitter release at a central synapse. Cell 79:717-727.

Giger RJ, Paster RJ, Heijnen S, Holtmaat AJ, Verhaagen J (1998) Anatomical distribution of the chemorepellant semaphorin III/ collapsin-1 in the adult rat and human brain. J Neurosci Res 52:27-42.

Hilbush BS, Morgan JI (1994) A third synaptotagmin gene, Syt3, in the mouse. Proc Natl Acad Sci USA 91:8195-8199.

Kingsbury AE, Gallo V, Woodhams PL, Balazs R (1985) Survival, morphology, and adhesion properties of cerebellar interneurons cultured in chemically defined and serum-supplemented medium. Brain Res 349:17-25.

Koibuchi N, Chin WW (2000) Thyroid hormone action and brain development. Trends Endocrinol Metab 11:123-128.

Legrand J (1979) Morphogenetic actions of thyroid hormones. Trends Neurosci 2:234-236.

Louis JC, Magal E, Muir D, Manthorpe M, Varon S (1992) CG-4, a new bipotential glial cell line from rat brain, is capable of differentiating in vitro into either mature oligodendrocytes or type-2 astrocytes. J Neurosci Res 31:193-204.

Mangelsdorf DJ, Thummel C, Beato M, Herrlich P, Schutz G, Umesono K, Blumberg B, Kastner P, Mark M, Chambon P, Evans RM (1995) The nuclear receptor superfamily: the second decade. Cell 83:835-839.

Morreale de Escobar G, Ruiz-Marcos A, Escobar del Rey F (1983) Thyroid hormone and the developing brain. In: Congenital hypothyroidism (Dussault JH, Walker P, eds), pp 85-126. New York: Marcel Dekker.

Nalefski EA, Falke JJ (1996) The C2 domain calcium-binding motif: structural and functional diversity. Protein Sci 5:2375-2390.

Nicholson JL, Altman J (1972a) The effects of early hypo- and hyperthyroidism on the development of rat cerebellar cortex. I. Cell proliferation and differentiation. Brain Res 44:13-23.

Nicholson JL, Altman J (1972b) Synaptogenesis in the rat cerebellum: effects of early hypo- and hyperthyroidism. Science 176:530-532.

Nordquist DT, Kozak CA, Orr HT (1988) cDNA cloning and characterization of three genes uniquely expressed in cerebellum by Purkinje neurons. J Neurosci 8:4780-4789.

Oppenheimer JH, Schwartz HL (1997) Molecular basis of thyroid hormone-dependent brain development. Endocr Rev 18:462-475.

Perin MS, Fried VA, Mignery GA, Jahn R, Südhof TC (1990) Phospholipid binding by a synaptic vesicle protein homologous to the regulatory region of protein kinase C. Nature 345:260-263.

Rabie A, Legrand J (1973) Effects of thyroid hormone and undernourishment on the amount of synaptosomal fraction in the cerebellum of the young rat. Brain Res 61:267-278.

Rami A, Patel AJ, Rabie A (1986) Thyroid hormone and development of the rat hippocampus: morphological alterations in granule and pyramidal cells. Neuroscience 19:1217-1226.

Rizo J, Südhof TC (1998) C domains, structure, and universal $\mathrm{Ca}^{2+}$ binding domain. J Biol Chem 273:15879-15882.

Samuels HH, Stanley F, Casanova J (1979) Depletion of L-3,5,3'triiodothyronine and L-thyroxine in euthyroid calf serum for use in cell culture studies of the action of thyroid hormone. Endocrinology 101:80-85

Schiavo G, Osborne SL, Sgouros JG (1998) Synaptotagmins: more isoforms than functions? Biochem Biophys Res Commun 248:1-8.

Schwartz HL (1983) Effect of thyroid hormone on growth and development. In: Molecular basis of thyroid hormone action (Oppenheimer JH, Samuels HH), pp. 413-444. New York: Academic.

Strait KA, Zou L, Oppenheimer JH (1992) $\beta 1$ isoform-specific regulation of a triiodothyronine-induced gene during cerebellar development. Mol Endocrinol 6:1874-1880.

Südhof TC, Rizo J (1996) Synaptotagmins: C2-domain proteins that regulate membrane traffic. Neuron 17:379-388.

Thompson CC (1996) Thyroid hormone-responsive genes in developing cerebellum include a novel synaptotagmin and a hairless homolog. J Neurosci 16:7832-7840.

Thompson CC, Bottcher MC (1997) The product of a thyroid hormoneresponsive gene interacts with thyroid hormone receptors. Proc Natl Acad Sci USA 94:8527-8532.

Thompson CC, Potter GB (2000) Thyroid hormone action in neural development. Cereb Cortex 10:939-945.

Ullrich B, Li C, Zhang JZ, McMahon H, Anderson RGW, Geppert M, Südhof TC (1994) Functional properties of multiple synaptotagmins in brain. Neuron 13:1281-1291.

Vician L, Lim IK, Ferguson G, Tocco G, Baudry M, Herschman HR (1995) Synaptotagmin IV is an immediate early gene induced by depolarization in PC12 cells and brain. Proc Natl Acad Sci USA 92:2164-2168.

Zhang J, Lazar MA (2000) The mechanism of action of thyroid hormones. Annu Rev Physiol 62:439-466. 